\title{
Robust LQ Control for Harmonic Reference/Disturbance Signals
}

\author{
Hakan Köroğlu $\quad$ Ömer Morgül \\ Bilkent University, Dept. Electrical \& Electronics Engineering, Bilkent, 06533, Ankara, Turkey. \\ Cor. Author: H. Köroğlu, Tel.: (90-312) 290-2384, Fax: (90-312) 266-4192, E-Mail: koroglu@ee.bilkent. edu.tr
}

\begin{abstract}
Linear Quadratic (LQ) controller design is considered for continuous-time systems with harmonic signals of known frequencies and it is shown that the design is reducible to an interpolation problem. All LQ optimal loops are parametrized by a particular solution of this interpolation problem and a (free) stable/proper transfer function. The appropriate choice of this free parameter for optimal stability robustness is formulated as a multiobjective design problem and reduced to a Nevanlinna-Pick interpolation problem with some interpolation points on the boundary of the stability domain. Using a related result from the literature, it is finally shown that, if there is sufficient penalization on the power of the control input, the level of optimum stability robustness achievable with LQ optimal controllers is the same as the level of optimum stability robustness achievable by arbitrary stabilizing controllers.
\end{abstract}

Keywords : Control design, LQ control, $\mathcal{H}_{\infty}$ control, Harmonic signals.

\section{Introduction}

Consideration of harmonic signals in control systems is important from theoretical as well as practical viewpoints. Theoretical motivation comes from the fact that harmonic signals can be expressed as the superposition of countably many sinusoids. This fact can be utilized to launch a frequency domain approach for the treatment of harmonic signals in feedback systems. On the other hand, many engineering systems experience harmonic disturbances. Two common examples are helicopters experiencing vibrations [1], and disk drives subjected to periodic disturbances [13]. The solutions of the standard control problems of reference tracking and disturbance rejection for deterministic signals utilize the (by now classical) Internal Model Principle of [5]. In accordance with this principle, the dynamics of the deterministic signal is replicated in the loop, and the overall design is formulated as the minimization of an appropriate LQ cost. This minimization can then be performed via the state-space methods available for $L Q$ design (see e.g. $[2,7])$. An LQ design problem with harmonic signals is considered only recently in [11], with a formulation similar to the original LQ problem, by defining the cost directly for the original system. The development is considered for multivariable discretetime systems and the optimal solutions are described by some interpolation conditions. A much simpler formulation of the LQ problem for harmonic signals was done in [9]. The present work extends the development of [9] to achieve some results which can be easily interpreted and employed in multiobjective design problems.

The rest of the paper is organized as follows. In the next section, we describe the setup and give the basic problem formulation of LQ control for harmonic signals (LQH control). In Section 3, we review the internal stability of linear time-invariant (LTI) single-input singleoutput (SISO) feedback control systems. In Section 4, we show that the problem of LQH control is reducible to an interpolation problem and parametrize all $\mathrm{LQH}$ optimal controllers by a particular solution of this interpolation problem and a free stable/proper transfer function. Motivated by this parametrization, we consider a multiobjective design problem in Section 5 and develop a robust controller synthesis procedure. After we present a simple example in the penultimate section, we conclude by summarizing our results.

\section{Problem Formulation}

Throughout the paper, we restrict our attention to SISO and LTI continuos-time systems, though the results are applicable to discrete-time systems with some minor modifications. We consider plants and controllers with real rational transfer functions. The minor notational preferences are as follows. The space of stable and proper rational functions, $H(s)$, is denoted by $\mathcal{H}_{\infty}$ and the well-known $\mathcal{H}_{\infty}$ norm is expressed via the standard notation: ||$H \|_{\infty}=\sup _{\omega}|H(j \omega)|$. Occasionally, we treat transfer functions as operators acting on time signals for notational convenience. The complex conjugate of $H(j \omega)$ is shown as $H^{*}(j \omega)$ and the relative degree of $H$ (degree of the denominator minus degree of the numerator) is denoted by $\rho_{H}$. 


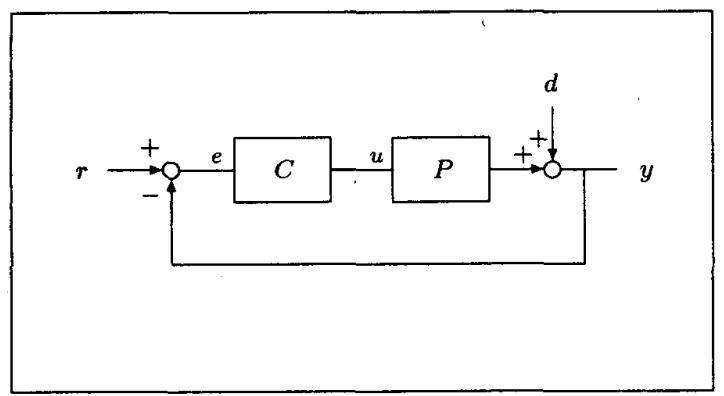

Figure 1: Unity feedback control system.

We consider the unity feedback configuration of Figure 1, where $P$ is the LTI plant and $C$ is the LTI controller. The external signals $r$ and $d$ are respectively the reference and the disturbance signals, wheras the internal signals $e(=r-y)$ and $u$ are respectively the tracking error and the control input. The infinite horizon frequency weighted LQ cost can be defined for SISO continuous-time systems as

$$
J_{\mathrm{LQ}} \triangleq \lim _{\tau \rightarrow \infty} \frac{1}{\tau} \int_{0}^{\tau}[e(t)]^{2}+[F(s) u(t)]^{2},
$$

where $F$ is a LTI stable and minimum phase filter. With the minimization of the $\mathrm{LQ}$ cost, the plant output is forced to follow the reference command quadratically optimally with appropriately low control effort. In this paper, we will study the LQ control problem with harmonic reference and disturbance signals. Harmonic signals can be expressed as

$$
x(t)=\sum_{i=1}^{n_{\omega}} a_{i} \cos \left(\omega_{i} t+\theta_{i}\right),
$$

where $\omega_{i}$ are the frequencies present in the signal and $a_{i}$ and $\theta_{i}$ are respectively the associated magnitudes and phases. In this setup, the problem of LQH control can be stated as follows:

Problem 1 [LQH Control] Given a LTI plant $P$ and the information that $r$ and $d$ are harmonic.signals with known frequencies (and possibly unknown magnitudes and phases), find a LTI feedback controller $C$ such that the feedback system of Figure 1 minimizes $J_{\mathrm{LQ}}$.

\section{Internal Stability Constraints}

For simplicity of presentation, we consider in the following parts of our paper that the plant $P$ has simple strict right half plane poles $p_{i} ; i=1, . ., n_{p}$ and simple right half plane zeros (excluding infinity) $z_{i} ; i=1, . ., n_{z}$. We will refer to $p_{i}$ as unstable poles and to $z_{i}$ as unstable zeros. With the well-known Blaschke products of poles and zeros are defined as

$$
B_{p}(s) \triangleq \prod_{i=1}^{n_{p}} \frac{s-p_{i}}{s+p_{i}^{*}}, B_{z}(s) \triangleq \prod_{i=1}^{n_{z}} \frac{s-z_{i}}{s+z_{i}^{*}},
$$

we can express $P$ as

$$
P(s)=B_{z}(s) B_{p}^{-1}(s) \tilde{P}(s),
$$

where $\tilde{P}$ is a stable and minimum-phase transfer function. The well known Youla parameter, sensitivity and the complementary sensitivity function of the closed loop system of Figure 1 are defined as

$$
\begin{aligned}
& Q(s) \triangleq[1+P(s) C(s)]^{-1} C(s), \\
& S(s) \triangleq[1+P(s) C(s)]^{-1}, \\
& T(s) \triangleq[1+P(s) C(s)]^{-1} P(s) C(s) .
\end{aligned}
$$

It is easy to find that $S$ and $T$ relate to $Q$ as

$$
\begin{aligned}
& S(s)=1-P(s) Q(s), \\
& T(s)=P(s) Q(s) .
\end{aligned}
$$

The system of Figure 1 is internally stable if and only if the functions $S, Q, P S$ and $T$ are all $\mathcal{H}_{\infty}$ (i.e. stable and proper) transfer functions [6]. It is well known that this internal stability requirement can equivalently be expressed as some interpolation constraints on the feedback system functions $Q, S$ and $T[3,4,6])$. The key observation at this point is the unacceptability of unstable pole/zero cancellations among $P$ and $C$. From this we can infer that $P C$ should interpolate to zero at $z_{i}$ and to infinity at $p_{i}$. It then follows that $Q\left(p_{i}\right)=0$, $S\left(p_{i}\right)=0$ and $T\left(z_{i}\right)=0$, or equivalently

$$
Q=B_{p} \tilde{Q}, S=B_{p} \tilde{S}, T=B_{z} \tilde{T},
$$

where $\tilde{Q}, \tilde{S}$ and $\tilde{T}$ are $\mathcal{H}_{\infty}$ transfer functions (see [3]). Noting that $\tilde{T}=\tilde{P} \tilde{Q}$ (from (4), (9) and (10)), and $S+T=1$ (from (6) and (7)), we can write

$$
B_{z}(s) \tilde{P}(s) \tilde{Q}(s)+B_{p}(s) \tilde{S}(s)=1 .
$$

This relation imposes on $\tilde{Q}$ a group of interpolation constraints which can be expressed as

$$
\tilde{Q}\left(p_{i}\right)=B_{z}^{-1}\left(p_{i}\right) \tilde{P}^{-1}\left(p_{i}\right) ; \quad \forall i ; i=1, . ., n_{p} .
$$

This way the problem of an internally stable system design is reduced to finding an $\mathcal{H}_{\infty}$ transfer function $\tilde{Q}$ which satisfies (12). After the construction of such a $\tilde{Q}$, equation (11) can be solved for $\tilde{S}$ and the feedback controller can be obtained from (5) and (6) (after some necessary cancellations) as the minimal realization of

$$
C(s)=\frac{\tilde{Q}}{\tilde{S}} .
$$




\section{LQ Design for Harmonic Signals as an Interpolation Problem}

With $x$ defined as

$$
x(t) \triangleq r(t)-d(t)
$$

the tracking error and the control input in the feedback system of Figure 1 are determined by

$$
\begin{aligned}
& u(t)=Q(s) x(t) \\
& e(t)=S(s) x(t),
\end{aligned}
$$

where the relations are expressed in operator notation. If the control system of Figure 1 is internally stable and $x$ is a quasi-stationary signal with spectrum $\Phi_{x x}$, the spectral input/output relations and Parseval's identity (see e.g. [12]) can be used to evaluate the LQ cost as

$$
J_{\mathrm{LQ}}=\frac{1}{2 \pi} \int_{-\infty}^{\infty} j_{\mathrm{LQ}}(\omega) \Phi_{x x}(\omega) d \omega
$$

where $j_{\mathrm{LQ}}$ can be found (after some straightforward manipulations) to be

$$
\begin{aligned}
j_{\mathrm{LQ}}(\omega)= & \left(|F|^{2}+|P|^{2}\right)\left|Q-P^{*}\left(|F|^{2}+|P|^{2}\right)^{-1}\right|^{2} \\
+|F|^{2}\left(|F|^{2}+|P|^{2}\right)^{-1} &
\end{aligned}
$$

If $x$ is a harmonic signal of the form (2), $\Phi_{x x}$ can be found as [12]

$$
\Phi_{x x}(\omega)=\frac{\pi}{2} \sum_{i=1}^{n_{\omega}} a_{i}^{2}\left[\delta\left(\omega-\omega_{i}\right)+\delta\left(\omega+\omega_{i}\right)\right] .
$$

where $\delta$ is the well-known Dirac's delta function. Using this in (17) and noting that we are working with real rational transfer functions, we can immediately obtain the expression of the LQH cost ( $J_{\mathrm{LQ}}$ with the $x$ of (2)) as

$$
J_{L Q H}=\frac{1}{2} \sum_{i=1}^{n_{\omega}} a_{i}^{2} j_{L Q}\left(\omega_{i}\right)
$$

Our preliminary result, stated in the following theorem, is a direct consequence of this expression.

Theorem 1 Let the control system of Figure 1 be internally stable and $x$ be a harmonic signal given by (2). The feedback loop is LQH optimal (minimizes $J_{\mathrm{LQH}}$ ) if and only if $\tilde{Q}$, defined according to (10), satisfies

$$
\tilde{Q}\left(j \omega_{i}\right)=\frac{B_{p}^{-1}\left(j \omega_{i}\right) P^{*}\left(j \omega_{i}\right)}{\left|F\left(j \omega_{i}\right)\right|^{2}+\left|P\left(j \omega_{i}\right)\right|^{2}} ; \quad \forall \omega_{i} ; i=1, . ., n_{\omega} .
$$

The optimal $L Q H$ cost is given by

$$
J_{\mathrm{LQH}}^{\mathrm{opt}}=\frac{1}{2} \sum_{i=1}^{n_{\omega}} \frac{a_{i}^{2}\left|F\left(j \omega_{i}\right)\right|^{2}}{\left|F\left(j \omega_{i}\right)\right|^{2}+\left|P\left(j \omega_{i}\right)\right|^{2}} .
$$

As understood from this theorem, the $L Q$ design for harmonic signals necessitates the determination of a stable/proper transfer function which satisfies the interpolation constraints of (21). It is then obvious that Problem 1 is equivalent to the following one.

Problem 2 [Equivalent Problem of LQH Control] Find a transfer function $\tilde{Q} \in \mathcal{H}_{\infty}$ which satisfies the interpolation constraints given by (12) and (21).

This problem can be solved by some standard methods. Given the interpolation data, a stable polynomial of appropriate degree (to satisfy the relative degree requirement) can be assigned as the denominator polynomial of $\tilde{T}$ and the problem can be reduced to a polynomial interpolation problem, which can be solved by -for example- Lagrange interpolation. Once the equivalent problem is solved, the corresponding controller can be obtained via (13). Obviously there are infinitely many solutions of the LQ design problem for harmonic signals. If $\tilde{Q}_{0}$ is a particular solution of the equivalent problem and $B_{\omega}$ is the Blaschke product of the harmonics defined as 1

$$
B_{\omega}(s) \triangleq \prod_{i=1}^{n_{\omega}} \frac{s^{2}+\omega_{i}^{2}}{(s+1)^{2}}
$$

then all LQH optimal controllers can be parametrized as

$$
C_{\mathrm{LQH}}(s)=\frac{\tilde{Q}_{0}(s)+B_{p}(s) B_{\omega}(s) H(s)}{\tilde{S}_{0}(s)-B_{z}(s) B_{\omega}(s) \tilde{P}(s) H(s)},
$$

where $H$ is an arbitrary $\mathcal{H}_{\infty}$ transfer function and $\tilde{S}_{0}$ is the minimal realization of $B_{p}^{-1}\left(1-B_{z} \tilde{P} \tilde{Q}_{0}\right)$. This parametrization describes the whole set of universally optimal (see [11]) LQH controllers. In other words, the controllers which can be expressed as in (24) optimize $J_{L Q}$ for any $x$ of the form (2), independent of the magnitudes $a_{i}$ and phases $\theta_{i}$.

\section{Robust LQ Design for Harmonic Signals}

The principal result of the previous section is the reduction of an $\mathrm{LQ}$ design problem to an interpolation problem. It is well known that some basic $\mathcal{H}_{\infty}$ design problems also reduce to interpolation problems (see e.g. [4]). As the LQ design problem of the previous section admits infinitely many solutions parametrized in (24), other design objectives can also be considered. Common framework of treatment with $\mathcal{H}_{\infty}$ control motivates us for multiobjective designs in which an $\mathcal{H}_{\infty}$ cost is to be minimized in addition to the LQH cost. Two

\footnotetext{
${ }^{1}$ If $\omega_{i}$ is zero, the corresponding term in the product should be replaced by $s /(s+1)$.
} 
basic problems of $\mathcal{H}_{\infty}$ control are the weighted sensitivity minimization problem and the stability robustness optimization problem [4]. We consider below the stability robustness optimization problem together with LQH minimization.

Stability robustness optimization (for additive plant perturbations) in an $\mathcal{H}_{\infty}$ setting considers the minimization of the cost

$$
J_{\mathcal{H}_{\infty}} \triangleq\|W(s) Q(s)\|_{\infty}
$$

where $W$ is a stable, proper and minimum phase transfer function (see [3]). This problem (as well as some other $\mathcal{H}_{\infty}$ control problems) is closely related with the Nevanlinna-Pick interpolation problem (see [4]). Nevanlinna-Pick interpolation problem (in its classical formulation) considers the determination of transfer functions in $\mathcal{H}_{\infty}$, which satisfy a group of interpolation constraints at given strict right half plane points and which have $\mathcal{H}_{\infty}$ norms less than or equal to unity. The stability robustness optimization problem reduces to such a problem as a result of the internal stability constraints imposed by the unstable poles. If we define $G$ as

$$
G(s) \triangleq W(s) \tilde{Q}(s),
$$

we can replace $J_{\mathcal{H}_{\infty}}{ }^{-}$with $\|G\|_{\infty}$, as we have $\left|B_{p}(j \omega)\right|=$ 1. It follows from (26) and (12) that $G \in \mathcal{I}_{p}$

$$
\mathcal{I}_{p} \triangleq\left\{H \in \mathcal{H}_{\infty}: H\left(p_{i}\right)=\alpha_{i} ; \forall i ; i=1, . ., n_{p} \cdot\right\}
$$

and $\alpha_{i}$ 's are defined as

$$
\alpha_{i} \triangleq W\left(p_{i}\right) B_{z}^{-1}\left(p_{i}\right) \tilde{P}^{-1}\left(p_{i}\right) ; i=1, . ., n_{p} .
$$

If the relative degree of $W$ is greater than zero, we should also impose $\rho_{G}=\rho_{W}$. Yet, independent of the relative degree constraint on $G$, the optimum value of $J_{\mathcal{H}_{\infty}}$ is equal to the maximum lower bound of the $\mathcal{H}_{\infty}$ norms of the transfer functions in $\mathcal{I}_{p}$, which we define as

$$
\gamma\left(\mathcal{I}_{p}\right) \triangleq \inf _{H \in \mathcal{I}_{p}}\|H\|_{\infty}
$$

It is well known from classical theory $[4,8]$ that $\gamma\left(\mathcal{I}_{p}\right)$ is given by

$$
\gamma\left(\mathcal{I}_{p}\right)=\sqrt{\dot{\lambda}_{\max }\left(\mathbf{A}^{-1} \mathbf{B}\right)}
$$

where $\mathbf{A}$ and $\mathbf{B}$ are the matrices defined as

$$
\mathbf{A} \triangleq\left[\frac{1}{p_{i}+p_{j}^{*}}\right] ; \mathbf{B} \triangleq\left[\frac{\alpha_{i} \alpha_{j}^{*}}{p_{i}+p_{j}^{*}}\right] ; i, j=1, \ldots, n_{p}
$$

and $\lambda_{\max }$ denotes the maximum eigenvalue. If we scale the interpolation data with $\gamma^{-1}\left(\mathcal{I}_{p}\right)$, we end up with the classical Nevanlinna-Pick interpolation problem, for which we have closed form solutions [8] as well as iterative construction algorithms $[3,4]$. The relative degree constraint leads to a technical hindrance in $\mathcal{H}_{\infty}$ optimization. For this reason, usually the optimum cannot be achieved by proper controllers. Yet it can be approached arbitrarily by an appropriate modification of the optimal (improper) controller [3,4].

The observation that $\mathcal{H}_{\infty}$ optimization can also be treated in the interpolation framework motivates us for the following multiobjective design problem:

Problem 3 [Robust LQH Control] Given a LTI plant with simple unstable poles and zeros having positive real parts, determine among the $L Q H$ optimal controllers of (24) the one which minimizes $\left\|W(s) Q_{\mathrm{LQH}}(s)\right\|_{\infty}$ for a stable, proper and minimum phase transfer function $W$.

Among the transfer functions $G \in \mathcal{I}_{p}$, the ones corresponding to an LQH optimal loop are in $\mathcal{I}_{\omega}$ where

$$
\mathcal{I}_{\omega} \triangleq\left\{H \in \mathcal{H}_{\infty}: H\left(j \omega_{i}\right)=\beta_{i} ; \forall i ; i=1, . ., n_{\omega}\right\}
$$

and $\beta_{i}$ 's are defined as

$$
\beta_{i} \triangleq \frac{W\left(j \omega_{i}\right) B_{p}^{-1}\left(j \omega_{i}\right) P^{*}\left(j \omega_{i}\right)}{\left|F\left(j \omega_{i}\right)\right|^{2}+\left|P\left(j \omega_{i}\right)\right|^{2}} ; i=1, . ., n_{\omega} .
$$

In other words, for LQH optimality we should have $G \in$ $\mathcal{I}_{p} \cap \mathcal{I}_{\omega}$. This means that we can state the equivalent problem of robust LQ control for harmonic signals as follows.

Problem 4 [Equivalent Problem of Robust LQH Control] Given a LTI plant with simple unstable poles/zeros having positive real parts, and a stable, proper and minimum phase transfer function $W$, find a transfer function $G \in \mathcal{I}_{p} \cap \mathcal{I}_{\omega}$ of relative degree $\rho_{G}=\rho_{W}$ such that $\|G\|_{\infty}$ is (arbitrarily close to its) minimum.

It follows from the discussion above that, the determination of the optimum cost necessitates the use of the Nevanlinna-Pick theory. Due to the presence of interpolation points on the boundary of the stability domain (which is the imaginary axis for our case), the standard theory (which assumes no boundary interpolation points) is not applicaple. An extended treatment of the Nevanlinna-Pick problem to include boundary interpolation points is done by Khargonekar and Tannenbaum in [8]. For the sake of completeness, we cite below their relevant result with necessary adaptations.

Theorem 2 ([8]) Let $p_{i}$ be distinct strict right plane points and $j \omega_{i}$ be distinct imaginary axis points. Let the sets (of transfer functions) $\mathcal{I}_{p}$ and $\mathcal{I}_{\omega}$ be defined by (27) and (32). The maximum lower bound for the $\mathcal{H}_{\infty}$ norms of the transfer functions in $\mathcal{I}_{p} \cap \mathcal{I}_{\omega}$ is given by

$$
\gamma\left(\mathcal{I}_{p} \cap \mathcal{I}_{\omega}\right)=\max _{i}\left\{\gamma\left(\mathcal{I}_{p}\right),\left|\beta_{i}\right|\right\}
$$


Application of this result to our case gives us the bound on the maximum attainable stability robustness together with LQ optimality for harmonic signals. The result also has an important implication concerning the simultaneous optimization of $J_{\mathrm{LQH}}$ and $J_{\mathcal{H}_{\infty}}$. Before we present this as a theorem, we define a new cost $J_{\mathrm{RLQH}}$ as

$$
J_{\mathrm{RLQH}} \triangleq J_{\mathrm{LQH}}+J_{\mathcal{H}_{\infty}} .
$$

The following theorem, which states an important result on the minimization of $J_{\mathrm{RLQH}}$, is a direct corollary of Theorem 2 .

Theorem 3 Let $P$ be a plant with simple unstable poles $\left(p_{i}\right)$ and zeros $\left(z_{i}\right)$ with positive real parts, and let $\mathcal{I}_{p}$ and $\gamma\left(\mathcal{I}_{p}\right)$ be defined as in (27) and (29). If $F$ satisfies

$$
\left|F\left(j \omega_{i}\right)\right| \geq\left|P\left(j \omega_{i}\right)\right|\left[\max \left\{0, \frac{\left|W\left(j \omega_{i}\right)\right|}{\gamma\left(\mathcal{I}_{p}\right)\left|P\left(j \omega_{i}\right)\right|}-1\right\}\right]^{1 / 2},
$$

for all $i=1, . ., n_{\omega}$, then the infimum of $J_{\mathrm{RLQH}}$ is given $b y \inf J_{\mathrm{RLQH}}=J_{\mathrm{LQH}}^{\mathrm{opt}}+\inf J_{\mathcal{H}_{\infty}}$.

Remark 1 Conditions given by (36) determine the best achievable level of tracking/rejection for a harmonic signal of known frequencies, if stability robustness optimization is the principal concern. It also shows the significance of the presence of the frequency weighting filter in the $L Q$ cost (which was mainly for the penalization of the power of the control input) from the aspect of stability robustness.

Obviously the optimum of our multiobjective design problem is generally unattainable due to the relative degree constraint and the degeneracy imposed by the interpolation conditions at the harmonics. Yet any (slightly) greater cost, which is arbitrarily close to the optimum, is achievable (similarly to the $\mathcal{H}_{\infty}$ optimization). We outline below a procedure, with which such solutions can be constructed.

Procedure 1 [Robust LQH Design] Given $P, F$, $W$ and $\omega_{i}$;

1. Determine $\gamma\left(\mathcal{I}_{p} \cap \mathcal{I}_{\omega}\right)$ using (30) and (34).

2. Find $G \in \mathcal{I}_{p} \cap \mathcal{I}_{\omega}$ such that $\|G\|_{\infty} \leq \gamma\left(\mathcal{I}_{p} \cap \mathcal{I}_{\omega}\right)+\epsilon$ with (arbitrarily small) $\epsilon>0$.

3. Modify $G$ as $G \rightarrow G\left(1-B_{p} B_{\omega}(\varepsilon s) /(\varepsilon s+1)\right)^{\rho_{W}}$ with (arbitrarily small) $\varepsilon>0$.

4. Set $\tilde{Q}_{\mathrm{RLQH}}=W^{-1} G$.

5. Set $\tilde{S}_{\mathrm{RLQH}}(s)$ as the minimal realization of $B_{p}^{-1}\left(1-B_{z} \tilde{P} \tilde{Q}_{\mathrm{RLQH}}\right)$.

6. Obtain $C_{\mathrm{RLQH}}$ as $C_{\mathrm{RLQH}}=\tilde{Q}_{\mathrm{RLQH}} / \tilde{S}_{\mathrm{RLQH}}$.
The second step of this procedure necessitates a Nevanlinna-Pick interpolation algorithm that can treat boundary interpolation points. Such an algorithm can be found in [10]. Actually, the algorithm of [10] can also cope with unity relative degree requirement, and this might be useful in obtaining low degree controllers. If $G$ is constructed to have a certain relative degree, the third step of the algorithm should be modified accordingly.

\section{Illustrative Design Example}

We illustrate the robust $L Q$ design with harmonic signals for a simple plant with transfer function $P=$ $1 /(s-1)$. Clearly, we have $B_{z}=1, B_{p}=(s-1) /(s+1)$ and $\tilde{P}=1 /(s+1)$. The internal stability constraint on $\tilde{Q}$ is simply $\tilde{Q}(1)=2$. With $W=(s+0.2) /(s+1)^{2}$, the infimum of $(25)$ is $W(1) \tilde{Q}(1)=0.6$ and thus the minimizing $G$ is simply 0.6 (see [4]). This solution cannot be achieved by any proper controller (as the required relative degree for $G$ is unity), yet can arbitrarily be approached. If robust LQH design is considered for frequencies $\omega_{i}=\{0.5,1,2\}$, conditions of (36) are (just) satisfied by $F(0.5 j)=0, F(j)=0.3177$ and $F(2 j)=0.3156$. Assuming a denominator polynomial of $(s+1)^{6}$ for $\tilde{Q}$, and finding the appropriate numerator polynomial to satisfy (12) and (21), we can obtain a particular LQH optimal controller as

$C_{\mathrm{LQH}}(s)=\frac{8.993 s^{6}+11.05 s^{5}+45.2 s^{4}+22.08 s^{3}+32.93 s^{2}+4.376 s+3.376}{s^{6}-0.9929 s^{5}+8.962 s^{4}-1.241 s^{3}+11.68 s^{2}-0.2482 s+2.376}$

Applying the procedure for robust LQH design (by making use of the algorithm of [10] with relative degree treatment), we can construct a robust LQH controller with $\epsilon=0.05$ as

$$
\begin{gathered}
C_{\mathrm{RLQH}}(s)=\frac{16.24 s^{8}+16.08 s^{7}+83.59 s^{6}+82.98 s^{5}+80.05 s^{4}+80.33 s^{3}}{s^{8}+9.994 s^{7}+5.419 s^{6}+52.25 s^{5}+5.439 s^{4}+52.14 s^{3}} \\
\ldots \frac{+14.3 s^{2}+14.74 s-0.0819}{+0.6084 s^{2}+9.926 s-0.1071}
\end{gathered}
$$

The magnitude variation of $W Q$ correponding to the optimally robust loop, LQH optimal loop and robust LQH optimal loop are are displayed in Figure 2. As the figure shows, $W Q_{\mathrm{RLQH}}$ has a similar magnitude variation with the $W Q_{\mathcal{H}_{\infty}}$ except at the harmonics and the high-pass band. The $\mathcal{H}_{\infty}$ norm of $W Q_{\mathrm{RLQH}}$ is 0.65 . If $C_{\mathrm{RLQH}}$ is constructed with a smaller $\epsilon$, the variations will look more similar and the $\mathcal{H}_{\infty}$ cost of $C_{\mathrm{RLQH}}$ will be closer to 0.6 , however the numerical sensitivity of the synthesis algorithm does not allow extremely small $\epsilon$ 's. Moreover, the results of the simulations performed with a harmonic disturbance and zero reference (see Figure 3) show that, the robust LQH controller has a poor transient behaviour (control is started after the second period). With smaller $\epsilon$ 's, the transient response gets worse. This is the most remarkable tradeoff in the robust design. 


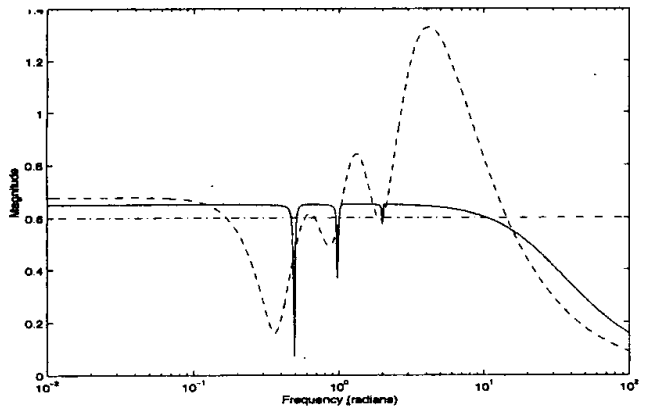

Figure 2: Magnitude variation of $W Q\left(W Q_{\mathrm{RLQH}}\right.$ : solid, $W Q_{\mathrm{LQH}}$ : dashed, $W Q_{\mathcal{H}_{\infty}}$ : dash-dotted).
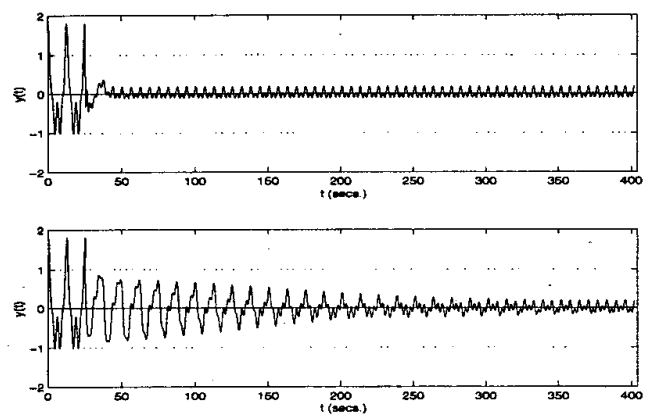

Figure 3: Simulation results with $\omega_{i}=\{0.5,1,2\}, a_{i}=$ $\{1,0.5,0.3\}$ and $\theta_{i}=0\left(C_{\mathrm{LQH}}\right.$ : top, $C_{\mathrm{RLQH}}$ : bottom).

\section{Conclusions}

We considered the LQ design problem for linear timeinvariant continuous-time systems with harmonic signals of known frequencies and showed that the design is reducible to an interpolation problem. We then parametrized all LQH optimal controllers in terms of a particular LQH optimal solution and a free parameter. The choice of this free parameter to obtain a desired overall closed-loop behaviour motivated a multiobjective design problem, in which an $\mathcal{H}_{\infty}$ cost as well as the LQH cost is to be minimized. Here we considered the stability robustness optimization together with LQ optimization for harmonic signals and showed that this problem is reducible to a Nevanlinna-Pick interpolation problem with some interpolation points on the boundary of the stability domain. If the frequency weighting filter in the LQ cost has sufficiently great magnitudes at the harmonics under consideration, the optimal robust stability level can arbitrarily be approached while simultaneously satisfying the LQH optimality conditions. This result finds its use in a design procedure in which stability robustness is the principal concern and tracking/rejection of the harmonic signals is the secondary aim. Our results apply to discrete-time systems with some minor modifications. Several other $\mathcal{H}_{\infty}$ design problems (e.g. weighted sensitivity minimization, gain margin optimization) can be considered together with LQ optimal design for harmonic signals and they can be solved via similar interpolation methods.
Acknowledgements: We would like to express our sincere thanks to Prof. Hitay Özbay and Prof. Peter Dorato for supplying us some references and commenting on our work.

\section{References}

[1] S. Bittani, F. Lorito, and S. Strada. An LQ approach to active control of vibrations in helicopters. ASME Journal of Dynamic Systems, Measurement, and Control, 118(3):482-488, 1996.

[2] E. J. Davison and P. Patel. Application of the robust servomechanism controller to systems with periodic tracking/disturbance signals. International Journal of Control, 47(1):111-127, 1988.

[3] P. Dorato, L. Fortuna, and G. Muscato. Robust Control for Unstructured Perturbations: An Introduction. Springer-Verlag, NY, 1992.

[4] J. C. Doyle, B. A. Francis, and A. R. Tannenbaum. Feedback Control Theory. Macmillan Publishing Company, NY, 1992.

[5] B. A. Francis and W. M. Wonham. The Internal Model Principle of control theory. Automatica, 12(5):457-465, 1976.

[6] J. W. Helton and O. Merino. Classical Control Using $\mathcal{H}_{\infty}$ Methods: Theory, Optimization, and Design. SIAM, NY, 1998.

[7] A. Iftar. Optimal solution to the servomechanism problem for systems with stochastic and deterministic disturbances. International Journal of Control, 51(6):1327-1341, 1990.

[8] P. P. Khargonekar and A. R. Tannenbaum. NonEuclidian metrics and the robust stabilization of systems with parameter uncertainty. IEEE Transactions on Automatic Control, AC-30(10):1005-1013, 1985.

[9] H. Köroğlu and Ö. Morgül. LQ optimal design at countably many frequencies. In Proceedings of the 37th IEEE Conference on Decision and Control, pages 1195-1196, Tampa, Florida, USA, December 1998.

[10] Y. Li. U-Parameter Design: Feedback System Design with Guaranteed Robust Stability. PhD thesis, Department of Electrical and Computer Engineering, University of New Mexico, February 1989.

[11] A. Lindquist and V. A. Yakubovich. Universal regulators for optimal tracking in discrete-time systems affected by harmonic disturbances. IEEE Transactions on Automatic Control, AC-44(9):1688-1704, 1999.

[12] L. Ljung. System Identification: Theory for the User. Prentice-Hall, Englewood Cliffs, NJ, 1987.

[13] A. Sacks, M. Bodson, and P. Khosla. Experimental results of adaptive periodic disturbance cancellation in a high performance magnetic disk drive. ASME Journal of Dynamic Systems, Measurement, and Control, $118(3): 416-424,1996$. 